This is an electronic reprint of the original article. This reprint may differ from the original in pagination and typographic detail.

\author{
Author(s): Räbinä, Jukka; Mönkölä, Sanna; Rossi, Tuomo; Markkanen, Johannes; Gritsevich, \\ Maria; Muinonen, Karri
}

Title: $\quad$ Controlled time integration for the numerical simulation of meteor radar reflections

Year: $\quad 2016$

Version:

Please cite the original version:

Räbinä, J., Mönkölä, S., Rossi, T., Markkanen, J., Gritsevich, M., \& Muinonen, K. (2016). Controlled time integration for the numerical simulation of meteor radar reflections. Journal of Quantitative Spectroscopy and Radiative Transfer, 178 (July), 295-305. doi:10.1016/j.jqsrt.2016.02.009

All material supplied via JYX is protected by copyright and other intellectual property rights, and duplication or sale of all or part of any of the repository collections is not permitted, except that material may be duplicated by you for your research use or educational purposes in electronic or print form. You must obtain permission for any other use. Electronic or print copies may not be offered, whether for sale or otherwise to anyone who is not an authorised user. 


\section{Author's Accepted Manuscript}

Controlled time integration for the numerical simulation of meteor radar reflections

Jukka Räbinä, Sanna Mönkölä, Tuomo Rossi, Johannes Markkanen, Maria Gritsevich, Karri Muinonen



PII: $\quad$ S0022-4073(15)30125-4

DOI: $\quad$ http://dx.doi.org/10.1016/j.jqsrt.2016.02.009

Reference: JQSRT5222

To appear in: Journal of Quantitative Spectroscopy and Radiative Transfer

Received date: 15 September 2015

Revised date: 25 December 2015

Accepted date: 8 February 2016

Cite this article as: Jukka Räbinä, Sanna Mönkölä, Tuomo Rossi, Johanne Markkanen, Maria Gritsevich and Karri Muinonen, Controlled time integratio for the numerical simulation of meteor radar reflections, Journal of Quantitativ Spectroscopy and Radiative Transfer http://dx.doi.org/10.1016/j.jqsrt.2016.02.009

This is a PDF file of an unedited manuscript that has been accepted fo publication. As a service to our customers we are providing this early version o the manuscript. The manuscript will undergo copyediting, typesetting, an review of the resulting galley proof before it is published in its final citable form Please note that during the production process errors may be discovered whic could affect the content, and all legal disclaimers that apply to the journal pertain 


\title{
Controlled time integration for the numerical simulation of meteor radar reflections
}

\author{
Jukka Räbinäa ${ }^{\mathrm{a}}$, Sanna Mönköläa,*, Tuomo Rossi ${ }^{\mathrm{a}}$, Johannes Markkanen ${ }^{\mathrm{b}}$, \\ Maria Gritsevich ${ }^{c, d, e}$, Karri Muinonen ${ }^{\text {b,c }}$ \\ ${ }^{a}$ University of Jyvaskyla, Department of Mathematical Information Technology, P.O. \\ Box 35 (Agora), FI-40014 University of Jyvaskyla, Finland \\ ${ }^{b}$ Department of Physics, P.O. Box 64, FI-00014 University of Helsinki, Finland \\ ${ }^{c}$ Finnish Geospatial Research Institute, P.O. Box 15, FI-02431 Masala, Finland \\ ${ }^{d}$ Dorodnicyn Computing Centre, Federal Research Center "Computer Science and \\ Control" of the Russian Academy of Sciences, 119333 Moscow, Russia \\ ${ }^{e}$ Institute of Physics and Technology, Ural Federal University, 620002 Ekaterinburg, \\ Russia
}

\begin{abstract}
We model meteoroids entering the Earth's atmosphere as objects surrounded by non-magnetized plasma, and consider efficient numerical simulation of radar reflections from meteors in the time domain. Instead of the widely used finite difference time domain method (FDTD), we use more generalized finite differences by applying the discrete exterior calculus (DEC) and non-uniform leapfrog-style time discretization. The computational domain is presented by convex polyhedral elements. The convergence of the time integration is accelerated by the exact controllability method. The numerical experiments show that our code is efficiently parallelized. The DEC approach is compared to the volume integral equation (VIE) method by numerical experiments. The result is that both methods are competitive in modelling non-magnetized plasma scattering. For demonstrating the simulation capabilities of the DEC approach, we present numerical experiments of radar reflections and vary parameters in a wide range.
\end{abstract}

Keywords: electromagnetic scattering, meteors, radar reflections, plasmatic objects, discrete exterior calculus, volume integral equation PACS: 41.20.Jb, 02.60.Cb

\footnotetext{
${ }^{*}$ Corresponding author

Email address: sanna.monkola@jyu.fi (Sanna Mönkölä)
} 


\section{Introduction}

We consider a meteoroid entering the Earth's atmosphere at a hypervelocity of $11-72 \mathrm{~km} / \mathrm{s}$. Through the interaction with atmospheric particles, the meteoroid produces a bright event called a meteor. Consequently, a dense mass of plasma surrounds the meteoroid and leaves a plasma trail, referred to as meteor plasma, which diffuses into the background atmosphere behind the meteor. Meteors have been a subject of intensive study over the last century; in particular, a lot of work has been devoted to collecting and interpreting data acquired by optical, and later by radar, seismic, and infrasound networks.

Meteor head echoes, caused by radio waves scattered from the dense mass of plasma surrounding the meteor body along its atmospheric trajectory, have been studied by high-power large-aperture (HPLA) radars since 1960s (see [1]). Modern HPLA observations of meteor head echoes were made not earlier than in the 1990s [2], using the radar systems of the European Incoherent Scatter Scientific Association (EISCAT), the Canadian Meteor Orbit Radar, the Jicamarca high-power large-aperture VHF radar, the $46.5 \mathrm{MHz}$ MU radar system near Shigaraki, Japan, the Southern Argentine Agile MEteor Radar (SAAMER), and other dedicated systems [3, 4, 5, 6]. Although the considered radar observation volumes are narrow, being elongated in the vertical direction, huge amounts of meteor head echoes have been detected over the past years of observations. Most of the events are faint with no alternative to be detected visually or with intensified video cameras. Thus, understanding the way in which a meteor reflects radio waves could present a unique possibility to determine the range, propagation angle, and velocity of the object based on radar detections.

By using numerical simulation techniques, it is possible to test how changes in the atmospheric conditions affect the meteor radar reflections and explain unexpected results in the measurements. To get reliable simulation outputs, appropriate models, relevant methods, and valid input information has to be defined carefully. The physics of meteor radar reflections is considered as electromagnetic scattering. The most simplified models consider meteors to be solid obstacles, but the more realistic models present the scatterer as a plasmatic obstacle (see, e.g., [7, 8, 9, 10]). In particular, we apply the model, in which the dielectric tensor is derived from the equation of motion pre- 
senting charged, non-magnetized cold plasma, as presented in [9]. By using this model, the dispersive media can be treated in the time domain without convolution. Thin, practically two-dimensional, layers of three-dimensional space are considered with the model in [11], and the model is simulated in the three-dimensional domain in [12]. Due to the recent developments in computing resources, it is possible to improve the accuracy of the numerical simulations or solve more demanding problems. Consequently, the methods for simulating three-dimensional meteor plasma dynamics are currently under active research [13]. Nevertheless, increasing the number of available CPUs and memory is not enough as the size of the problem does not scale at the same pace as the available resources. Modern and efficient numerical methods offer advantages over the earlier approaches and more gain than the increase in computing power.

The traditional way of solving electromagnetic problems, presented in the space-time domain as partial differential equations, is to use the finitedifference time-domain method (FDTD) introduced by Yee [14]. This method is applied to ground penetrating radar simulations in [15], and meteor simulations in the two-dimensional domain in [11] and in the three-dimensional domain in [12]. The models of electromagnetic wave propagation in magnetized plasma have been recently solved in the three-dimensional domain with the FDTD method in [16, 17]. The other methods, used to discretize the models presenting the interaction between electromagnetic waves and plasma, include the continuous and discontinuous Galerkin approaches (see, e.g. [18]). Instead of applying the FDTD or another well-known method, we present a more genaralized numerical scheme for 3D simulations. Our approach is more flexible with respect to spatial and temporal discretizations than the FDTD method.

In this study, we present the scattering problem in terms of differential forms (see, e.g., [19]), and discrete exterior calculus (DEC) is used for spatial discretization [20]. Accordingly, the discrete spaces and exact differential operators mimic their continuous counterparts, preserving the physical properties of the problem. With the DEC framework, we use high-quality mesh structures that mimick the geometry of the close packing in crystal lattices [21]. Combined with non-uniform leapfrog-style time stepping, this approach gives significant savings in computing time compared to conventional methods. To get even more accurate results, we tailor the discretization formulas for time-harmonic plane-wave solutions, by including so-called harmonic corrections, to reduce the error of the solution into fractions compared 
to the traditional Yee method [21].

For solving time-harmonic problems, the simulations based on space-time models need to be continued until the steady-state solution is reached. Essentially, the procedure is computationally inefficient, but we control the time integration, by the exact controllability method [22], to accelerate the convergence towards the steady-state solution. The idea of exact controllability has been recently applied to the fields of acoustics [23, 24], elastodynamics [25], and electromagnetics [26], but this is the first time it is applied to three-dimensional meteor plasma dynamics. In the numerical experiments, we compare the method with the volume integral equation (VIE) formulation for the electric current [27].

The rest of this paper is organized as follows: First, we review the physical background and mathematical formulation of the model in Sec. 2. In Sec. 3, we present the numerical methods applied for solving the problem. For discretization we present the discrete exterior calculus and a non-uniform time discretization. The controlled time integration is presented as a minimization problem which is solved by a conjugate gradient method. The validation of the methods is provided by numerical experiments in Sec. 4. Finally, in Sec. 5, we present the concluding remarks.

\section{Model}

Based on the observations conducted by the different radar systems and post-processing techniques, there exist several models for meteor head echo simulations, (see, e.g. [28]). One reason for this is differences in the characteristics of the radar systems, e.g., in terms of frequency and antenna geometry, see [29]. According to the observations reported by, e.g., [30] and [31], the head echo can be modeled as over-dense scatter from a plasma layer surrounding the meteoroid, with a specific density distribution. In these models, the plasmatic object is assumed to be a conducting spherical object, and the electromagnetic phenomenon can be presented by partial differential equations coupling the electric and magnetic fields.

In [11], the dielectric tensor is derived from the equation of motion presenting charged plasma. By following it, and the principles presented in [32], solid obstacles surrounded by non-magnetized plasma can be modeled with 
the use of differential equations as

$$
\begin{aligned}
\varepsilon \frac{\partial \mathbf{E}}{\partial t}-\nabla \times \mathbf{H} & =-\sigma \mathbf{E}-\mathbf{J} \\
\mu \frac{\partial \mathbf{H}}{\partial t}+\nabla \times \mathbf{E} & =-\sigma^{*} \mathbf{H} \\
\frac{\partial \mathbf{J}}{\partial t}+\nu \mathbf{J} & =\varepsilon \omega_{p}^{2} \mathbf{E}
\end{aligned}
$$

where $\mathbf{E}=\left(\mathrm{E}_{1}, \mathrm{E}_{2}, \mathrm{E}_{3}\right)^{T} \in \mathbb{R}^{3}$ is the electric field, $\mathbf{H}=\left(\mathrm{H}_{1}, \mathrm{H}_{2}, \mathrm{H}_{3}\right)^{T} \in \mathbb{R}^{3}$ is the magnetic field, $\mathbf{J}=\left(\mathrm{J}_{1}, \mathrm{~J}_{2}, \mathrm{~J}_{3}\right)^{T} \in \mathbb{R}^{3}$ is the plasma current density, $\varepsilon$ is the electric permittivity, $\mu$ is the magnetic permeability, $\nu$ is the collision frequency, $\sigma$ is the electric conductivity, $\sigma^{*}$ is the magnetic conductivity, and $\omega_{p}$ is the plasma angular frequency.

The unbounded domain of the scattering problem is truncated to a finite computational domain by an absorbing boundary condition or layer. In this study, we use the Silver-Müller boundary condition (see, e.g., [33]) and the perfectly matched layer (PML). The unphysical PML is the state-of-the-art layer for this purpose. We apply the uniaxial PML [34], which is modified for the DEC, as explained in [35]. The PML restricts the edge elements with the condition that they must be orthogonal or parallel to the absorbing direction.

\section{Numerical method}

For computing a numerical solution for the problem, the computational domain is discretized to vertices, edges, faces, and volumes. Since the metricfree nature of differential forms allows the presentation of the variables as integrands over curves, surfaces, and volumes, we use such a presentation. That is, for instance, instead of the vector presentation $\mathbf{E}=\left(\mathrm{E}_{1}, \mathrm{E}_{2}, \mathrm{E}_{3}\right)$ for the electric field, we present a 1-form $\mathrm{E}_{1} d x_{1}+\mathrm{E}_{2} d x_{2}+\mathrm{E}_{3} d x_{3}$, where $d$ is the exterior derivative and $\left(x_{1}, x_{2}, x_{3}\right)^{T} \in \mathbb{R}^{3}$ (see, e.g. [36]). At the discrete level, we use the discrete exterior calculus (DEC) framework, applied to the transient Maxwell equations in [21]. For accelerating the time evolution of the simulation, we use a controlled time integration approach [37] via the exact controllability approach, which leads to the time-harmonic solution.

\subsection{Discrete exterior calculus}

We consider the computational domain as a complex of cell elements, such that we operate the three-dimensional computation on 1-cells (edges) and 2cells (faces). We use a pair of primal and dual meshes, where each primal 


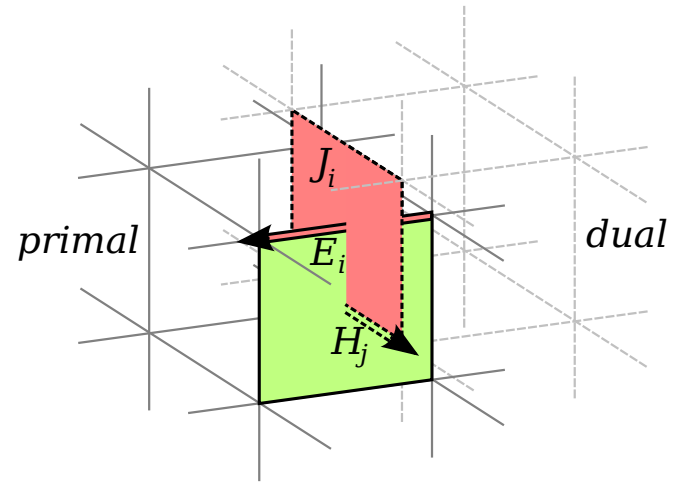

Figure 1: Spatial discretization is based on primal and dual meshes, where corresponding cells are orthogonal. $E, H$ and $J$ are discrete differential forms on primal edges, dual edges, and dual faces, respectively.

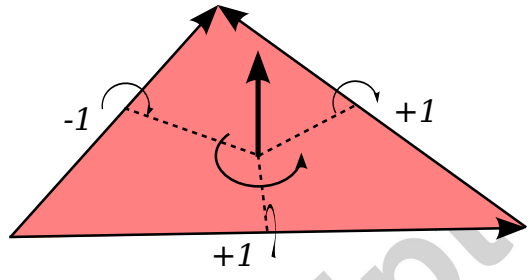

Figure 2: The incidence matrix $d_{1}$ indicates the rotation of an edge around the face (or a dual edge around the dual face).

$k$-cell, $\mathrm{k}=1,2$, corresponds to a dual $(3-k)$-cell. The $i$ :th edge is denoted by $\mathcal{E}_{i}, i=1, \ldots, n_{\mathcal{E}}$, and the $j$ :th face is denoted by $\mathcal{F}_{j}, j=1, \ldots, m_{\mathcal{F}}$. The corresponding dual elements are denoted by $\mathcal{F}_{i}^{*}$ and $\mathcal{E}_{j}^{*}$. The discrete differential forms $E$ (1-form), $H$ (1-form), and $J$ (2-form) are presented as vectors, for which each term is defined as (see Fig. 1)

$$
E_{i}:=\int_{\mathcal{E}_{i}} \mathbf{E} \cdot d \ell, \quad H_{j}:=\int_{\mathcal{E}_{j}^{*}} \mathbf{H} \cdot d \ell, \quad J_{i}:=\int_{\mathcal{F}_{i}^{*}} \mathbf{J} \cdot d a .
$$

That is, the discrete 1-forms are associated to the edges, and the discrete 2 -forms are associated to the faces.

The discrete counterparts of the curl operator are presented by the incidence matrix $d_{1}$ and its transpose $d_{1}^{T}$, such that,

$$
\int_{\mathcal{F}_{j}} \nabla \times \mathbf{E} \cdot d a=\left(d_{1} E\right)_{j}, \quad \int_{\mathcal{F}_{i}^{*}} \nabla \times \mathbf{H} \cdot d a=\left(d_{1}^{T} H\right)_{i} .
$$

Essentially, the incidence matrix represents the neighboring relations and relative orientations of the primal edges and faces (see Fig. 2). The entries, $\left(d_{1}\right)_{j, i}$, are non-zero if and only if the edge $\mathcal{E}_{i}$ is included in the boundary of the face $\mathcal{F}_{j}$. Further, the non-zero entries have the value +1 or -1 , indicating the relative orientation defined by counter-clockwise circulation. 
The physical character of the discretization is presented by the discrete Hodge star operator, $\star$, defining a mapping from a discrete 1-form to a discrete 2 -form, at the discrete level. The discrete Hodge star of a tensor $\alpha$ on cells $\mathcal{E}$ and $\mathcal{F}$ is defined as

$$
\star(\alpha, \mathcal{E}, \mathcal{F}):=\frac{|\mathcal{F}|}{|\mathcal{E}||\mathcal{F} \diamond \mathcal{E}|} \int_{\mathcal{F} \diamond \mathcal{E}} \alpha \mathbf{n}_{\mathcal{E}} \cdot \mathbf{n}_{\mathcal{E}} d v,
$$

where $\mathbf{n}_{\mathcal{E}}$ is the unit orientation vector of edge $\mathcal{E}$, and $\mathcal{F} \diamond \mathcal{E}$ is a convex hull including both $\mathcal{E}$ and $\mathcal{F}$. The discrete Hodge operators are introduced as diagonal matrices. The diagonal structure of the matrices is based on the orthogonality of the primal and dual elements. Applying the harmonic correction for both spatial and time discretization, the diagonal terms of the discrete Hodge operators are written as

$$
\begin{array}{rlrl}
\star \epsilon_{i, i} & =\star\left(\epsilon, \mathcal{E}_{i}, \mathcal{F}_{i}^{*}\right)\left[\kappa_{i} \frac{\phi_{i}}{\sin \phi_{i}}\right], & \star \sigma_{i, i} & =\star\left(\sigma, \mathcal{E}_{i}, \mathcal{F}_{i}^{*}\right)\left[\kappa_{i} \frac{1}{\cos \phi_{i}}\right], \\
\star \mu_{j, j}=\star\left(\mu, \mathcal{E}_{j}^{*}, \mathcal{F}_{j}\right)\left[\kappa_{j}^{*} \frac{\phi_{j}^{*}}{\sin \phi_{j}^{*}}\right], & \star \sigma_{j, j}^{*}=\star\left(\sigma^{*}, \mathcal{E}_{j}^{*}, \mathcal{F}_{j}\right)\left[\kappa_{j}^{*} \frac{1}{\cos \phi_{j}^{*}}\right],
\end{array}
$$

where the terms in square brackets indicate the harmonic correction. If these terms are equal to one, the Hodge terms are of Yee's kind. As shown in [21] and [35], the spatial correction terms, $\kappa_{i}$ and $\kappa_{j}^{*}$, can be computed by

$$
\begin{aligned}
& \kappa_{i}=\left(\frac{1-\frac{\kappa_{\mathcal{F}^{*}}}{5}+\frac{\kappa_{\mathcal{F}^{*}}^{2}}{56}}{1-\frac{\kappa_{\mathcal{F}^{*}}}{10}-\frac{\kappa_{\mathcal{E}}}{120}+\frac{\kappa_{\mathcal{F}^{*}}^{2}}{280}+\frac{\kappa_{\mathcal{F}} \kappa_{\mathcal{E}}}{1680}+\frac{\kappa_{\mathcal{\mathcal { V }}}^{2}}{22400}}\right), \\
& \kappa_{j}^{*}=\left(\frac{1-\frac{\kappa_{\mathcal{F}}}{5}+\frac{\kappa_{\mathcal{F}}^{2}}{56}}{1-\frac{\kappa_{\mathcal{F}}}{10}-\frac{\kappa_{\mathcal{E}}}{120}+\frac{\kappa_{\mathcal{F}}^{2}}{280}+\frac{\kappa_{\mathcal{F}} \kappa_{\mathcal{E}^{*}}}{1680}+\frac{\kappa_{\mathcal{\mathcal { E }}}^{2}}{22400}}\right),
\end{aligned}
$$

where $\kappa_{\mathcal{E}}=\omega^{2} \epsilon \mu\left|\mathcal{E}_{i}\right|^{2}, \kappa_{\mathcal{F}^{*}}=\omega^{2} \epsilon \mu r_{\mathcal{F}_{i}^{*}}^{2}, \kappa_{\mathcal{E}^{*}}=\omega^{2} \epsilon \mu\left|\mathcal{E}_{j}^{*}\right|^{2}$, and $\kappa_{\mathcal{F}}=\omega^{2} \epsilon \mu r_{\mathcal{F}_{j}}^{2}$. The face radius square $r_{\mathcal{F}}^{2}$ is computed using the inner radius $r^{\text {min }}$ and outer radius $r^{\max }$ (see Fig. 3), having

$$
r_{\mathcal{F}}^{2}=\frac{1}{n} \sum_{k=1}^{n}\left[\frac{2}{3}\left(r_{k}^{m i n}\right)^{2}+\frac{1}{3}\left(r_{k}^{\max }\right)^{2}\right] .
$$

The global time step size $\Delta t$ can be divided into smaller time steps using integer numbers $s_{\mathcal{E}_{i}}$ and $s_{\mathcal{E}_{j}^{*}}$ for each cell $\mathcal{E}_{i}$ and $\mathcal{E}_{j}^{*}$, respectively (see Fig. 4). 


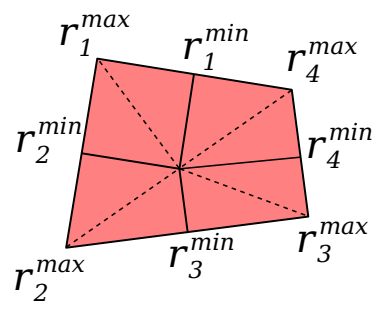

Figure 3: For an irregular quadrilateral, $r$ is replaced by the mean value computed from the distances $r_{j}^{\min }$ and $r_{j}^{\max }, j=1, \ldots, 4$.

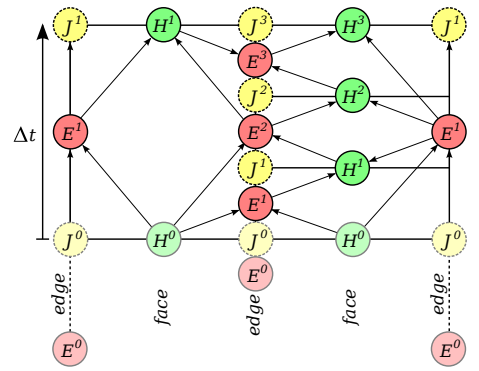

Figure 4: In non-uniform time stepping, $\Delta t$ is divided into smaller steps when necessary. The circles with numbers inside represent time instances of $E_{i}, H_{j}$ and $J_{i}$. The arrows illustrate the chronological order of the computation.

The time stepping is synchronized by selecting the numbers, $s_{\mathcal{E}_{i}}$ and $s_{\mathcal{E}_{j}^{*}}$, such that they can be written as powers of three, i.e., $s=3^{u} \in\{1,3,9,27, \ldots\}$. The harmonic corrections for time discretization are applied by using

$$
\phi_{i}=\frac{\omega \Delta t}{2 s_{\mathcal{E}_{i}}}, \quad \phi_{j}^{*}=\frac{\omega \Delta t}{2 s_{\mathcal{E}_{j}^{*}}},
$$

and the update formulas for the unknown variables are

$$
\begin{aligned}
& E_{i} \leftarrow E_{i}+\left(\frac{\star \epsilon_{i, i}}{\Delta t / s_{\mathcal{E}_{i}}}+\frac{\star \sigma_{i, i}}{2}\right)^{-1}\left[\left(d_{1}^{T} H\right)_{i}-\star \sigma_{i, i} E_{i}-J_{i}\right], \\
& H_{j} \leftarrow H_{j}+\left(\frac{\star \mu_{j, j}}{\Delta t / s_{\mathcal{E}_{j}^{*}}}+\frac{\star \sigma_{j, j}^{*}}{2}\right)^{-1}\left[-\left(d_{1} E\right)_{j}-\star \sigma_{j, j}^{*} H_{j}\right], \\
& J_{i} \leftarrow J_{i}+2\left(\frac{\omega}{\sin \phi_{i}}+\frac{\nu}{\cos \phi_{i}}\right)^{-1}\left[\star\left(\epsilon \omega_{p}^{2}, \mathcal{E}_{i}, \mathcal{F}_{i}^{*}\right) \kappa_{i} E_{i}-\frac{\nu}{\cos \phi_{i}} J_{i}\right] .
\end{aligned}
$$

The computational complexity of the method is of the order $\mathcal{O}(m N)$, where $N$ is the number of unknowns and $m$ is the number of time steps required to solve the system.

\subsection{Controlled time integration}

In principle, the time-harmonic solution can be reached by simple time integration (asymptotic approach), but we accelerate the convergence rate by 
applying a control. The basic idea of controllability is to steer a dynamical system from an initial state to a particular state by using an appropriate control mechanism. The concept of controllability can be divided into two categories, exact and approximate controllability. Approximate controllability makes it possible for the system to achieve a final state that belongs to an arbitrary small neighborhood of the final state, whereas exact controllability enables forcing the system from a preassigned initial state to a specific final state. Propagation, observation, and control issues of wave equations are reviewed by Zuazua in [38].

We apply the exact controllability technique pioneered by Bristeau, Glowinski, and Périaux [22] to time evolution. Essentially, the approach is a controlled variation of the asymptotic approach with periodic constraints, in which the time-dependent equation is simulated in time until the timeharmonic solution is reached. A natural quadratic error functional is based on the energy of the problem

$$
\mathcal{E}(E, H, J)=\frac{1}{2}\left(E^{T} \star \epsilon E+H^{T} \star \mu H+J^{T}\left(\star \epsilon \omega_{p}^{2}\right)^{-1} J\right) .
$$

From this basis, the time-harmonic solution can be found by minimizing $\mathcal{E}\left(E-E_{0}, H-H_{0}, J-J_{0}\right)$, where the initial conditions $E_{0}, H_{0}$, and $J_{0}$ are also the control variables. A quadratic functional can be minimized by solving the corresponding linear system with the conjugate gradient (CG) method (see, e.g., [39]). In principle, the gradient is computed at each CG iteration by solving two time-dependent equations, the state equation advancing forward in time and the corresponding adjoint state equation advancing backward in time. Only the current and previous gradient and search vectors and scalarvalued weights must be stored at each iterations. To guarantee the smooth initial approximation for the CG algorithm satisfying also the boundary conditions, we use the transition procedure suggested by Mur [40]. The residual of the algorithm, which defines at each iteration how far the solution is from a periodic solution, is also used to accelerate the convergence rate by giving a controlling impulse to the system. The discrete quadratic functional, which we minimize, is spanned by a diagonal mass matrix, and the algorithm operating in $L^{2}$-type Hilbert space does not need preconditioning.

\section{Numerical experiments}

We present numerical experiments considering the entry of a meteoroid into the Earth's atmosphere and study the scattering properties of the meteor 
plasma. Both the full scattering cross section and the backscattering cross section are computed, and the computing time and efficiency of the method is considered. The numerical method is implemented in $\mathrm{C}++$ programming language and parallelized using message passing interface (MPI) routines.

The meteor is viewed from the ground by a radar, as schematically illustrated in Fig. 5. The angle between the radar and the meteor propagation direction is denoted by $\alpha$. The considered computational domain, for simulating a bow shock around the meteoroid and surrounded area, is an origin-centered cylinder with a length of $10 \mathrm{~m}$ and diameter of $4 \mathrm{~m}$. In the meteor plasma trail, the collision frequency $\nu$ is $10 \mathrm{MHz}$ and the peak plasma frequency $f_{p}$ is $420 \mathrm{MHz}$. The angular plasma frequency $\omega_{p}$ is computed from the peak plasma frequency by the following distribution:

$$
\omega_{p}=2 \pi f_{p} F(x) \frac{r^{2}}{\left(r+k\left(x_{0}-x\right)\right)^{2}} e^{-\frac{y^{2}+z^{2}}{\left(r+k\left(x_{0}-x\right)\right)^{2}}},
$$

where

$$
F(x)=\left\{\begin{array}{lr}
e^{\frac{x-x_{0}}{l}}, & x \in]-\infty, x_{0},[, \\
1-\frac{\left(x-x_{0}\right)^{2}}{r^{2}}, & x \in\left[x_{0}, x_{0}+r\right], \\
0, & x \in] x_{0}+r, \infty[.
\end{array}\right.
$$

The constants of the plasma distribution are $r=0.6 \mathrm{~m}, k=0.15 \mathrm{~m}, l=3 \mathrm{~m}$ and $x_{0}=4 \mathrm{~m}$. The cross section of the distribution is illustrated in Fig. 6 . With a time-harmonic assumption, the plasma frequency and collision frequency can be turned into permittivity by the Drude model [41]. Since the peak plasma frequency exceeds the wave frequency, the permittivity becomes smaller than zero, which means the problem may become unstable, for example with the discrete-dipole approximation (DDA) solvers [42, 43].

\subsection{Parallelization test}

In the first test, the wavelength is $1.0 \mathrm{~m}$, implying that the radar frequency $f_{r}$ is approximately $300 \mathrm{MHz}$. The incident wave is circularly polarized. One of the simplest ways to construct cylinder-shaped geometry is to create a two-dimensional circular grid on the $y$-z-plane and stretch the grid through the $x$-direction to obtain a three-dimensional grid. The twodimensional base grid is mostly constructed of equilateral triangles, which fill the circle of radius $r=2 \mathrm{~m}$. The plasma region is surrounded by a $1 \mathrm{~m}$ thick layer, which is filled with quadrilateral elements provided with the PML 


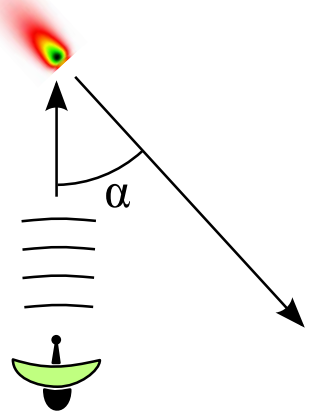

Figure 5: Meteor propagation angle $\alpha$.

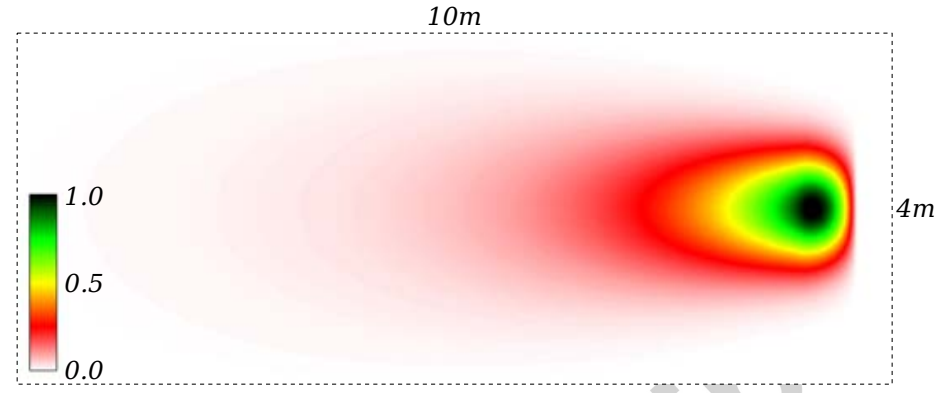

Figure 6: The normalized distribution of the meteor plasma frequency on the $x$-y-plane in a $10 \mathrm{~m} \times 4 \mathrm{~m}$ rectangle.

Table 1: Computing resources.

\begin{tabular}{cccc}
\hline machine name & processors & available cores & used cores \\
\hline Paasikivi & Intel E7-8837 @ 2.67GHz & 64 unreserved & 32 \\
Taito & Intel E5-2670@2.60GHz & 448 & 256 \\
Sisu & Intel E5-2690v3 @ 2.60GHz & 19,200 & 1,024 \\
\hline
\end{tabular}

condition and bounded by the Silver-Müller boundary condition. Thus, the resulting three-dimensional grid includes prism-shaped elements and twisted cubic elements. The average length of an edge of the grid is about $\frac{1}{20} \mathrm{~m}$, and the number of unknowns in the problem is 24,270,626.

For the parallelization, the mesh elements, which are nodes, edges, faces and bodies, are separated into blocks, and each block builds a computational domain for a computer core. To minimize the number of communications between processes, the surface area between the blocks should be kept small. The separation is applied by first ordering average positions of elements in the $x$-direction, and separating the elements by the median into two blocks. To obtain four blocks, the same procedure is repeated for both halves separately. The ten divisions needed for 1,024 cores are applied by ordering the elements in $x-, x-, y-, z-, z-, y-, x-, x-, y$-, $z$-directions, respectively. In this procedure, each core processes approximately the same number of elements, but the number of computing operations is not controlled. The mesh and the blocks are illustrated in Fig. 7.

The parallelization is tested by running the simulation over 100 time periods on three different supercomputers: Paasikivi, which is located at the 


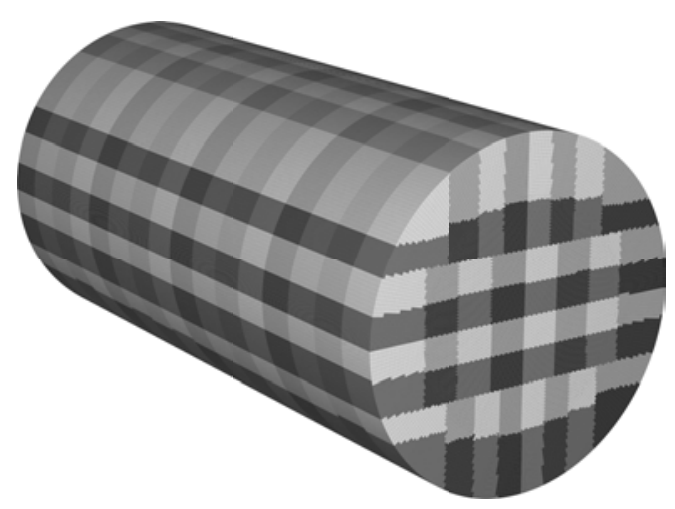

Figure 7: The mesh is divided into 1,024 blocks for the parallel computation.

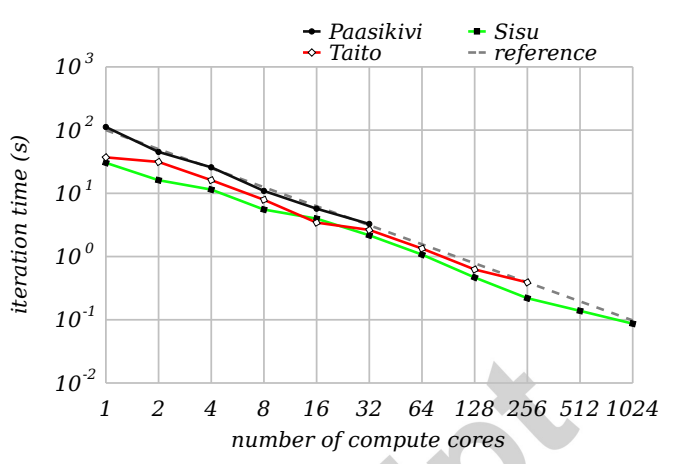

Figure 8: The iteration time by number of computer cores $n$. The reference line obeys function $t=100 \mathrm{~s} / n$.

University of Jyväskylä, and Taito and Sisu, which are computing resources of the CSC - IT Center. The details of the machines are presented in Table 1. The average simulation time for one time period (iteration time) is reported as the wall-clock time in seconds in Fig. 8. The reference line indicates the perfect parallelization, where the simulation time is inversely proportional to the number of cores. The simulation results demonstrate that the applied parallelization is efficient. The number of compute cores times the iteration time is between $87 \mathrm{~s}$ and $112 \mathrm{~s}$ on Paasikivi simulations, between $37 \mathrm{~s}$ and 100 s on Taito simulations, and between 30 and 89 on Sisu simulations. On Sisu, the iteration time is $30.4 \mathrm{~s}$ with a single processor and less than 0.087 s with 1,024 cores.

\subsection{Simulations with different parameters}

In this section, we vary the variables of geometry and the meteor model, and consider their effect on the backscattering properties. The mesh under the plasma region is filled with a so-called C15 grid [44]. That is, the primal grid is constructed by tetrahedra, and there are sixteen 12-hedra and eight 16-hedra in the dual grid structure. Such a construction is found to be a high quality grid for the DEC with good isotropic properties [21]. A half-meterthick layer, consisting of prism-shaped and twisted cubic elements, bounded by the Silver-Müller boundary condition, is built outside the region (see Fig. 9). There are two reasons for applying the layer: first, the near-to-far field computation, computed on the inner surface of the layer, can be applied with a smaller error. Second, the additional space between the target object 


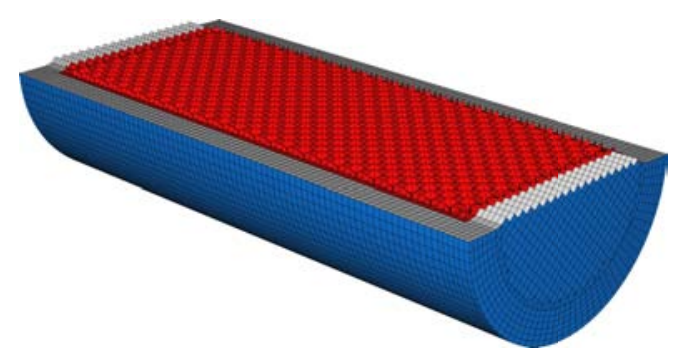

Figure 9: A half-cut illustration of the mesh used in DEC simulations with discretization level 10 elements per meter.

and the outer surface decreases the error caused by the absorbing boundary condition.

We apply radar frequencies of $160 \mathrm{MHz}, 224 \mathrm{MHz}, 300 \mathrm{MHz}, 422 \mathrm{MHz}$, $500 \mathrm{MHz}, 720 \mathrm{MHz}$, and $930 \mathrm{MHz}$, which are the AITAIR and EISCAT frequencies $[45,46]$. For the two highest radar frequencies, $720 \mathrm{MHz}$ and 930 $\mathrm{MHz}$, we use 28 elements per meter, while for the other frequencies we apply 20 elements per meter. The number of unknowns in the considered problems are 24,861,538 and 9,089,226, respectively. The solver is initialized by a transition stage [40], taking 10 time periods, for smoothly running the righthand-side values, presented in Eqs. (1)-(3), from zero to their known electric and magnetic source values. The controlled conjugate gradient iterations are run until the norm of the residual decreases down to $10^{-4}$. From each simulation, we compute three backscattering quantities: backscattering cross section $S_{11}$, same-circular cross section $S C=S_{11}+S_{44}$, and opposite-circular cross section $O C=S_{11}-S_{44}$, all of which are important physical observables in radar applications. In particular, the circular-polarization ratio $\frac{S C}{O C}$ is found to provide the best indications of the wavelength-scale geometric complexity of the material [47, 48].

First, we consider the effect of the meteor propagation angle $\alpha$ by varying it from 0 degrees (meteor moving towards the radar) up to 180 degrees (meteor moving away from the radar) with 5 degree increments. Second, we vary radar frequency and consider its effects on the backscattering properties. The results are shown in Fig. 10 and Fig. 11. Both variables have substantial and non-monotonic effects on the backscattering properties. Thus, the effects of these variables must be modeled when analyzing meteor properties using radar measurements. 


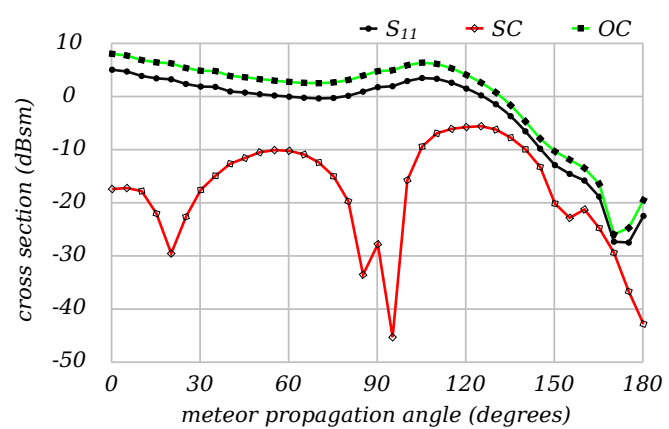

Figure 10: Cross sections as a function of the meteor propagation angle $\alpha$.

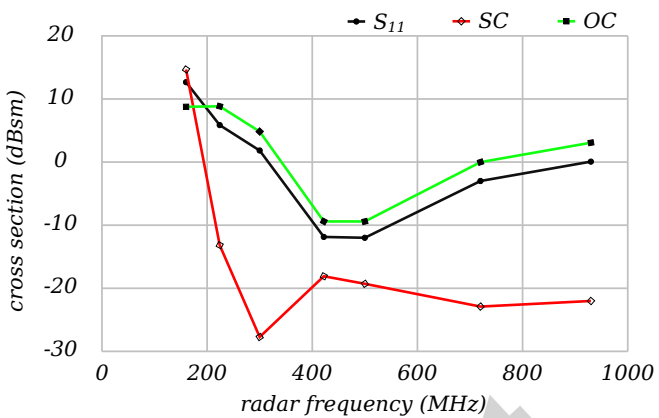

Figure 11: Cross sections as a function of radar frequency $f_{r}$.

Third, we consider the collision frequency $\nu$ describing the number of collisions between the particles in the ionosphere. Since the collision frequency is temperature-dependent, having different values depending on the time of day, we vary its value over a wide range, from $0.1 \mathrm{MHz}$ to $1,000 \mathrm{MHz}$. The collision frequency dramatically affects convergence during iterations; when $\nu \leq 1 \mathrm{MHz}$, the convergence is slow. However, the collision frequency seems to have only a small effect on the backscattering properties as shown in Fig. 12. The effect of the peak plasma frequency $f_{p}$ is much more considerable. We applied seven different peak plasma frequencies, $70 \mathrm{MHz}, 140$ $\mathrm{MHz}, 280 \mathrm{MHz}, 420 \mathrm{MHz}, 560 \mathrm{MHz}, 720 \mathrm{MHz}$, and $900 \mathrm{MHz}$. The results are illustrated in Fig. 13. The larger the plasma frequency, the larger the backscattering cross sections. The maximum difference between the smallest and the largest value is around $50 \mathrm{~dB}$. We also consider the effect of the meteor radius on the backscattering properties. By varying the parameter $r$ in Eqs. (5)-(6) from 0.1 to $0.8 \mathrm{~m}$, the results show that a larger radius implies larger cross sections, as illustrated in Fig. 14.

During the high-velocity atmospheric entry, a meteoroid may fragment into smaller pieces with a certain statistical mass distribution (see, e.g. [49]). In order to show how sensitive the model is with respect to the fragmentation of a meteor body, we consider the splitting of the meteoroid into two equal parts. We assume that the meteor radius is $r=0.3 \mathrm{~m}$ and the distance between the halves is denoted by $d$. We model the plasma trail of each half such that the peak plasma frequency is divided by $\sqrt{2}$. Both halves then have a $296.98 \mathrm{MHz}$ peak plasma frequency. The conductivity terms, $\epsilon \omega_{p}^{2}$, of the overlapping plasma distributions are then summed up in a way that the model of distance $d=0$ equals the single meteor model with peak plasma 


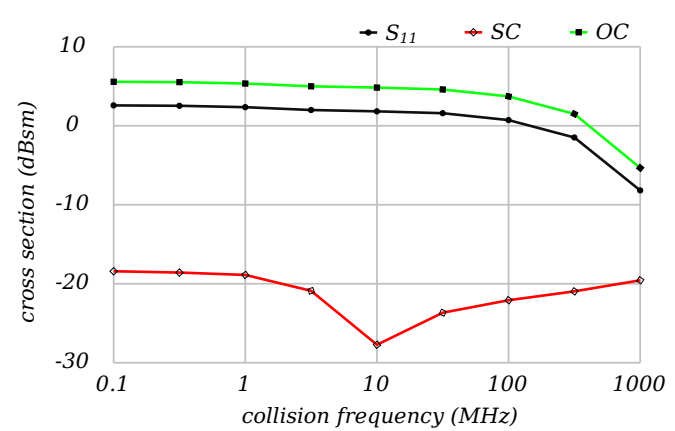

Figure 12: Cross sections as a function of collision frequency $\nu$.

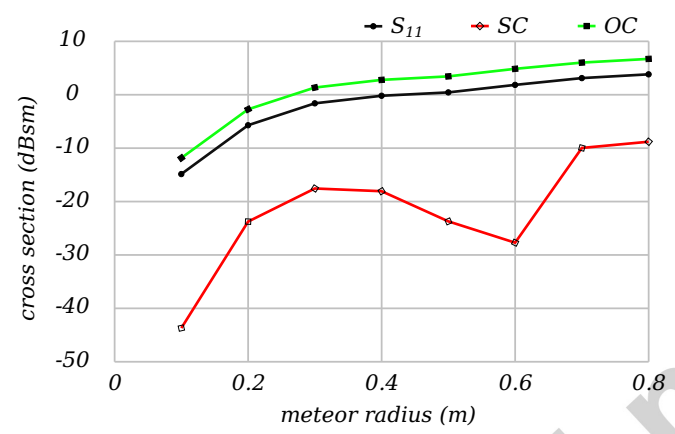

Figure 14: Cross sections as a function of meteor radius $r$.

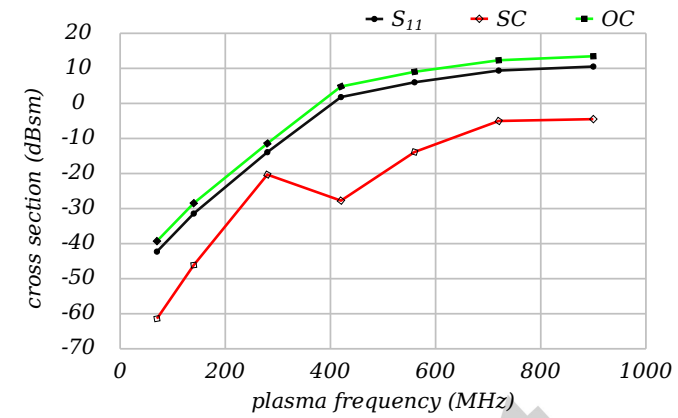

Figure 13: Cross sections as a function of peak plasma frequency $f_{p}$.

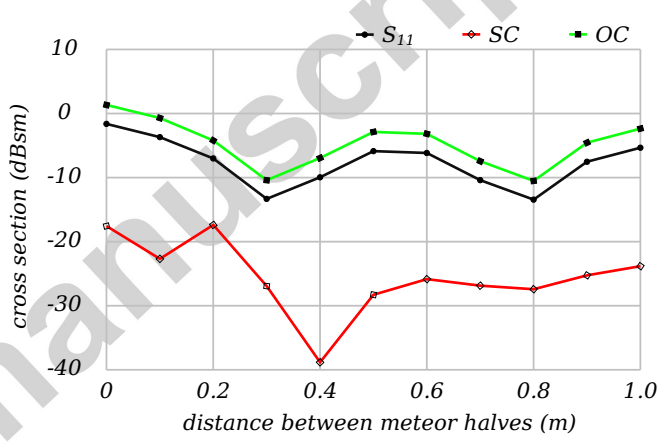

Figure 15: Cross sections of meteor pairs as a function of distance $d$ between meteors.

frequency of $420 \mathrm{MHz}$. We vary the distance $d$ between 0 and $1 \mathrm{~m}$ in 10 $\mathrm{cm}$ increments; the scattering results are illustrated in Fig. 15. Here, one can see the influence of destructive interference, since backscattering has the smallest values at distances $0.3 \mathrm{~m}$ and $0.8 \mathrm{~m}$.

\subsection{Comparison with the volume integral equation method}

In this section, we compare the simulation efficiency of the method with a volume integral equation (VIE) method. The scattering problem, described by the partial differential equations, can be reformulated as an equivalent system of integral equations. The volume integral equation (VIE) method for the electric current, (J-VIE) [27], is used in this paper. In this formulation, the unknown equivalent electric current density is expanded with piecewise constant basis functions. We apply Galerkin's procedure, in which identical basis and testing functions are used for converting the continuous equation 


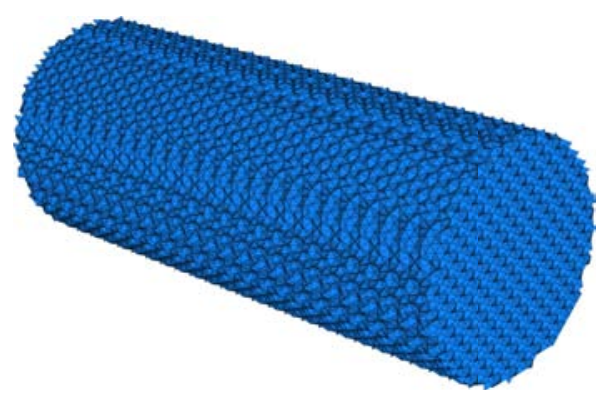

Figure 16: The mesh for VIE simulations with discretization level 10 elements per meter.

into a discrete set of linear equations [50]. The resulting system is solved iteratively by the generalized minimal residual method restarted after every 50 iterations (GMRES(50)), see, e.g., [39]. The matrix-vector multiplication in each iteration step is accelerated by the precorrected-FFT algorithm [51]. The most memory demanding data structure is the precorrection matrix. The memory requirement grows at each GMRESiteration, until the method is restarted, since the results from previous iterations need to be stored. Hence, the computational complexity of the method is of the order $\mathcal{O}(M N \log N)$, where $N$ is the number of unknowns and $M$ is the number of iterations required to solve the system.

Since the VIE solver requires no additional simulation domain outside the target object, the computational grid for it is simply a cut of the C15 grid, such that all volume element average positions are inside a cylinder of a $10 \mathrm{~m}$ length and $4 \mathrm{~m}$ diameter (see Fig. 16). With the DEC, we use the same mesh as in Sec. 4.2 (see Fig. 9). We simulate the problem with several discretization levels by applying grids with approximately 5, 7, 10 , 14, 20, 28, and 40 elements per meter. The simulations were run on CSC's supercomputer Taito with 16, 32, 64 or 128 computer cores, depending on the discretization level. We compared the methods with the peak plasma frequencies, $f_{p}, 280 \mathrm{MHz}$ and $420 \mathrm{MHz}$. With the VIE, the computing was stopped for $f_{p}=280 \mathrm{MHz}$ with a tolerance limit of $10^{-4}$, which took 10 iterations, and for $f_{p}=420 \mathrm{MHz}$ with a tolerance limit of $10^{-3}$, which took 200 iterations. For the DEC-based method, the norm of the residual $10^{-4}$ was used as a stopping criterion, and the average number of iterations was 66 for $f_{p}=280 \mathrm{MHz}$ and 403 for $f_{p}=420 \mathrm{MHz}$.

As a result of these simulations, we compute the Mueller matrices in- 


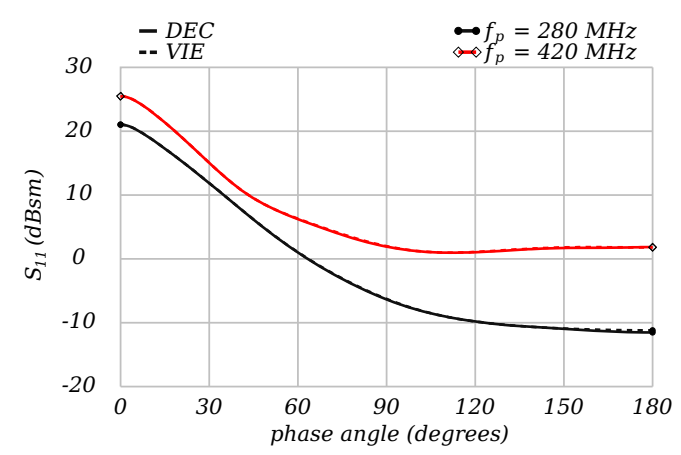

Figure 17: Phase functions at the maximum discretization levels.

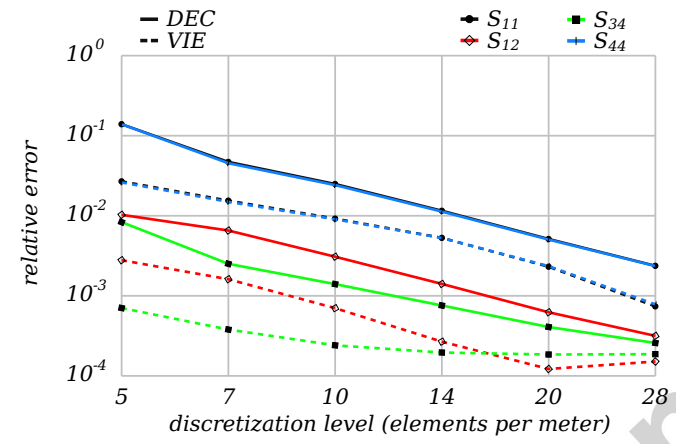

Figure 18: Relative errors of the Mueller matrix components in a test case $f_{p}=280$ $\mathrm{MHz}$.

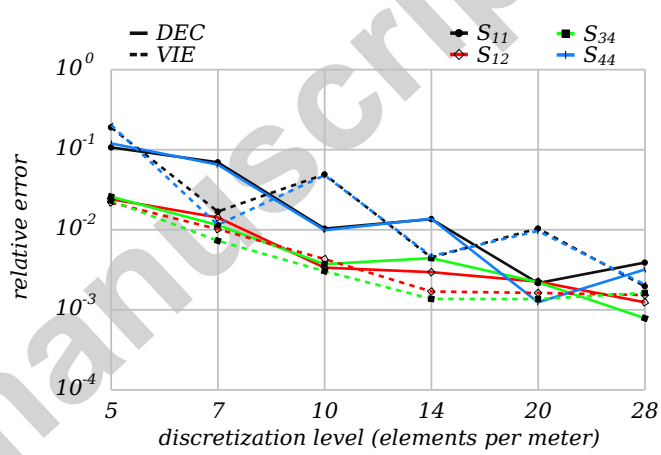

Figure 19: Relative errors of the Mueller matrix components in a test case $f_{p}=420$ $\mathrm{MHz}$.

tegrated over the azimuth angles. The accuracy of the simulation result is measured by the average relative error $\delta$ of the Mueller matrix component $S_{i j}$ with the formula

$$
\delta_{i j}=\frac{\int_{0}^{\pi}\left|S_{i j}(\theta)-S_{i j}^{\mathrm{ref}}(\theta)\right| d \theta}{\int_{0}^{\pi} S_{11}^{\mathrm{ref}}(\theta) d \theta} .
$$

In particular, we consider elements $S_{11}, S_{12}, S_{34}$, and $S_{44}$. The exact result of the problem is not known, so we compute the reference solution $S_{i j}^{\text {ref }}$ by taking the average of the Mueller matrices obtained by both methods with the finest discretization level of 40 . The relative error at the discretization level of 40 is then found to be approximately $0.1 \%$. Thus, the results of different methods are very close to each other, as shown in Fig. 17.

The relative errors of the $S$-components are illustrated with respect to the discretization levels in Fig. 18 and Fig. 19. The first case $\left(f_{p}=280 \mathrm{MHz}\right)$ is 
computationally less demanding, since the plasma frequency does not exceed the radar frequency. The results indicate that the errors decrease with the element size, and that VIE produces more accurate results compared to DEC at the same discretization level. The second case $\left(f_{p}=420 \mathrm{MHz}\right)$ includes region, where the plasma frequency is higher than the radar frequency. This makes the problem computationally much more demanding, and the convergence of relative error is non-monotonic with both methods. The reason for this phenomenon might be that the small details in discretization at the neighborhood of the meteor center can produce a remarkable difference in the result. Thus, a simulation with unfortunate element locations can produce a relatively large error. In the first test, the VIE gives more accurate results which might be because the stopping criteria of the methods are defined in a different way. In the second case, the accuracy of VIE and DEC are of the same order.

We have reported the computing times for solving the time-harmonic problem (processing time), as the wall-clock time in seconds multiplied by the number of processors used, in Fig. 20. The most remarkable difference between these two methods is that the initialization of VIE is highly demanding, and most of the total time is spent during the initialization. That is because a precorrection matrix for the FFT is assembled during the initialization. The initialization with the DEC takes only about $1 \%$ of the VIE initialization time. Essentially, for large number of unknowns, $N$, the number of iterations for the both methods as well as the number of time-steps for the DEC-based method are small compared to the number of unknowns. Thus, the asymptotic time complexity for the DEC method, $\mathcal{O}(N)$, is smaller than the asymptotic time complexity for the VIE method, $\mathcal{O}(N \log N)$. In the numerical tests, the DEC method seems to be at least an order of magnitude faster than the VIE method. Since the stopping criteria of the methods does not provide the same accuracy, we present the relative error of the Mueller matrix component $S_{11}$ with respect to simulation time in Fig. 21, and see that the DEC method is more efficient. That is because with DEC discretization, only diagonal matrices need to be inverted during time stepping. The performance of the DEC method does not strongly depend on the values of the material parameters, while the VIE method would require preconditioning to maintain a fast convergence rate for different parameters. 


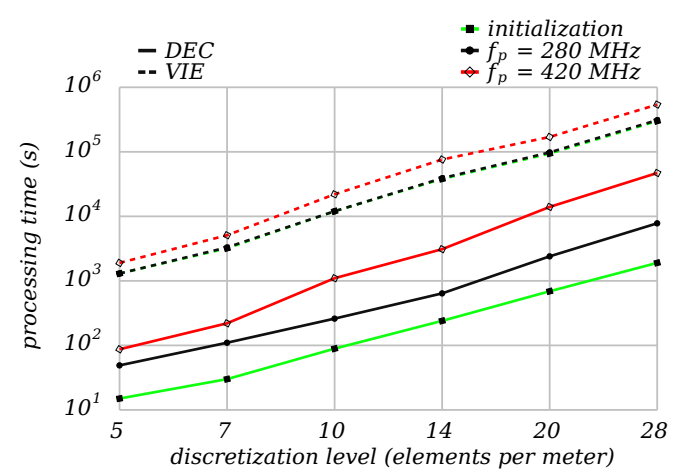

Figure 20: Processing times (s) for initialization and total simulation.



Figure 21: Relative error of component $S_{11}$ by the simulation processing time.

\section{Conclusions}

We have presented three-dimensional numerical simulations of radar reflections from meteors. The model was based on the assumption that meteors are solid objects surrounded by non-magnetized plasma. The spatial discretization by discrete exterior calculus and partly-structured non-uniform polyhedral grids, asynchronous leapfrog-style time discretization, and time integration accelerated by the exact controllability method, provided computational efficiency for solving time-harmonic problems. By numerical experiments, we validated the approach and compared it with the volume integral equation method for electric currents. DEC-based controlled time integration seems to be more efficient, and its performance is not sensitive to the level of discretization and the values of the material parameters.

\section{Acknowledgements}

This project has been funded by the Academy of Finland, grants 259925, 257966, 260027, and 260076, the ERC Advanced Grant No 320773, "Scattering and Absorption of Electromagnetic Waves in Particulate Media" (SAEMPL), and the Act 211 of the Government of the Russian Federation, agreement No 02.A03.21.0006. The authors acknowledge being a part of the network supported by the COST Action MP1104, "Polarization as a tool to study the Solar System and beyond" and by the COST Action TD1403 "Big Data Era in Sky and Earth Observation". 
[1] J. V. Evans, Radio-echo studies of meteors at 68-centimeter wavelength, Journal of Geophysical Research 70 (21) (1965) 5395-5416.

[2] A. Pellinen-Wannberg, G. Wannberg, Meteor observations with the european incoherent scatter UHF radar, Journal of Geophysical Research: Space Physics 99 (A6) (1994) 11379-11390.

[3] J. L. Chau, R. F. Woodman, F. Galindo, Sporadic meteor sources as observed by the Jicamarca high-power large-aperture VHF radar, Icarus 188 (1) (2007) 162-174.

[4] J. Jones, P. Brown, K. Ellis, A. Webster, M. Campbell-Brown, Z. Krzemenski, R. Weryk, The Canadian meteor orbit radar: system overview and preliminary results, Planetary and Space Science 53 (4) (2005) 413421.

[5] J. Kero, C. Szasz, T. Nakamura, D. D. Meisel, M. Ueda, Y. Fujiwara, T. Terasawa, K. Nishimura, J. Watanabe, The 2009-2010 MU radar head echo observation programme for sporadic and shower meteors: radiant densities and diurnal rates, Monthly Notices of the Royal Astronomical Society 425 (1) (2012) 135-146.

[6] R. Michell, D. Janches, M. Samara, J. Hormaechea, C. Brunini, I. Bibbo, Simultaneous optical and radar observations of meteor head-echoes utilizing SAAMER, Planetary and Space Science 118 (2015) 95-101.

[7] V. C. Liu, On ionospheric aerodynamics, Progress in Aerospace Sciences 16 (3) (1975) 273-297.

[8] L. Foschini, On the interaction of radio waves with meteoric plasma, arXiv preprint astro-ph/9804279.

[9] L. J. Nickisch, P. M. Franke, Finite-difference time-domain solution of Maxwell's equations for the dispersive ionosphere, IEEE Antennas and Propagation 34 (5) (1992) 33-39.

[10] B. Chakraborty, Principles of plasma mechanics, New Age International, 2007.

[11] L. Dyrud, D. Wilson, S. Boerve, J. Trulsen, H. Pecseli, S. Close, C. Chen, Y. Lee, Plasma and Electromagnetic Simulations of Meteor Head Echo 
Radar Reflections, Advances in Meteoroid and Meteor Science, Springer New York, 2008, pp. 383-394.

URL http://dx.doi .org/10.1007/978-0-387-78419-9_54

[12] Y. Yu, J. J. Simpson, An EJ collocated 3-D FDTD model of electromagnetic wave propagation in magnetized cold plasma, IEEE Transactions on Antennas and Propagation 58 (2) (2010) 469-478.

[13] M. M. Oppenheim, Y. S. Dimant, First 3-d simulations of meteor plasma dynamics and turbulence, Geophysical Research Letters 42 (3) (2015) 681-687.

[14] K. S. Yee, Numerical solution of initial boundary value problems involving Maxwell's equations in isotropic media, IEEE Transactions on antennas and propagation 14 (3) (1966) 302-307.

[15] M. Restano, G. Picardi, R. Seu, 1D-FDTD characterization of ionosphere influence on ground penetrating radar data inversion, Antennas and Propagation, IEEE Transactions on 62 (4) (2014) 2223-2230.

[16] B. T. Nguyen, C. Furse, J. J. Simpson, A 3-D stochastic FDTD model of electromagnetic wave propagation in magnetized ionosphere plasma, IEEE Transactions on Antennas and Propagation 63 (1) (2015) 304-313.

[17] A. Samimi, J. J. Simpson, An efficient 3-D FDTD model of electromagnetic wave propagation in magnetized plasma, Antennas and Propagation, IEEE Transactions on 63 (1) (2015) 269-279.

[18] P. Li, L. J. Jiang, Simulation of electromagnetic waves in the magnetized cold plasma by a DGFETD method, IEEE Antennas and Wireless Propagation Letters 12 (2013) 1244-1247.

[19] H. Cartan, Differential Forms, Kershaw Publishing Company, London, 1971.

[20] M. Desbrun, A. N. Hirani, M. Leok, J. E. Marsden, Discrete exterior calculus, preprint, arXiv:math/0508341v2 [math.DG] (2005).

[21] J. Räbinä, S. Mönkölä, T. Rossi, Efficient time integration of Maxwell's equations by generalized finite-differences, SIAM Journal on Scientific Computing 37 (2015) B834-B854, http://dx.doi.org/10.1137/ 140988759. 
[22] M. O. Bristeau, R. Glowinski, J. Périaux, Controllability methods for the computation of time-periodic solutions; Application to scattering, Journal of Computational Physics 147 (2) (1998) 265-292.

[23] E. Heikkola, S. Mönkölä, A. Pennanen, T. Rossi, Controllability method for the Helmholtz equation with higher-order discretizations, Journal of Computational Physics 225 (2) (2007) 1553-1576.

[24] S. Kähkönen, R. Glowinski, T. Rossi, R. Mäkinen, Solution of timeperiodic wave equation using mixed finite-elements and controllability techniques, Journal of Computational Acoustics 19 (4) (2011) 335-352.

[25] S. Mönkölä, E. Heikkola, A. Pennanen, T. Rossi, Time-harmonic elasticity with controllability and higher order discretization methods, Journal of Computational Physics 227 (11) (2008) 5513-5534.

[26] J. Räbinä, S. Mönkölä, T. Rossi, A. Penttilä, K. Muinonen, Comparison of discrete exterior calculus and discrete-dipole approximation for electromagnetic scattering, Journal of Quantitative Spectroscopy \& Radiative Transfer 146 (2014) 417-423.

[27] J. Markkanen, P. Ylä-Oijala, A. Sihvola, Discretization of volume integral equation formulations for extremely anisotropic materials, IEEE Trans. Ant. and Propag. 60 (2012) 5195-5202.

[28] L. Dyrud, D. Janches, Modeling the meteor head echo using Arecibo radar observations, Journal of Atmospheric and Solar-Terrestrial Physics 70 (13) (2008) 1621-1632.

[29] J. Kero, C. Szasz, T. Nakamura, T. Terasawa, H. Miyamoto, K. Nishimura, A meteor head echo analysis algorithm for the lower VHF band, Annales Geophysicae 30 (4) (2012) 639-659.

URL http: //www. ann-geophys .net/30/639/2012/

[30] L. E. Vertatschitsch, J. D. Sahr, P. Colestock, S. Close, Meteoroid head echo polarization features studied by numerical electromagnetics modeling, Radio Science 46 (6) (2011) - RS6016.

[31] G. Wannberg, A. Westman, A. Pellinen-Wannberg, Meteor head echo polarization at $930 \mathrm{MHz}$ studied with the EISCAT UHF HPLA radar, 
Annales Geophysicae 29 (6) (2011) 1197-1208.

URL http: //www . ann-geophys .net/29/1197/2011/

[32] H. L. Pécseli, Waves and oscillations in plasmas, CRC Press, 2012.

[33] P. Monk, Finite element methods for Maxwell's equations, Oxford University Press, 2003.

[34] S. D. Gedney, An anisotropic perfectly matched layer-absorbing medium for the truncation of FDTD lattices, IEEE Transactions on Antennas and Propagation 44 (12) (1996) 1630-1639.

[35] J. Räbinä, On a numerical solution of the Maxwell equations by discrete exterior calculus, Dissertation (Ph.D.), University of Jyväskylä., http: //urn.fi/URN : ISBN : 978-951-39-5951-7 (2014).

[36] K. F. Warnick, P. H. Russer, Differential forms and electromagnetic field theory, Progress in Electromagnetics Research 148 (2014) 83-112.

[37] D. Pauly, T. Rossi, Theoretical considerations on the computation of generalized time-periodic waves, Advances in Mathematical Sciences and Applications 21 (1) (2011) 105-131.

[38] E. Zuazua, Propagation, observation, and control of waves approximated by finite difference methods, SIAM Review 47 (2) (2005) 197-243.

[39] Y. Saad, Iterative Methods for Sparse Linear Systems, 2nd edition, SIAM, Philadelphia, 2003.

[40] G. Mur, The finite-element modeling of three-dimensional electromagnetic fields using edge and nodal elements, IEEE Transactions on Antennas and Propagation 41 (7) (1993) 948-953.

[41] P. Drude, Zur elektronentheorie der metalle, Annalen der Physik 306 (3) (1900) 566-613.

[42] O. S. Vartia, P. Ylä-Oijala, J. Markkanen, S. Puupponen, A. Seppälä, A. Sihvola, T. Ala-Nissila, On the applicability of discrete dipole approximation for plasmonic particles, Journal of Quantitative Spectroscopy and Radiative Transfer 169 (2016) 23-35. 
[43] M. A. Yurkin, A. G. Hoekstra, The discrete-dipole-approximation code ADDA: capabilities and known limitations, Journal of Quantitative Spectroscopy and Radiative Transfer 112 (13) (2011) 2234-2247.

[44] J. B. Friauf, The crystal structures of two intermetallic compounds, Journal of the American Chemical Society 49 (12) (1927) 3107-3114.

[45] S. Close, M. Oppenheim, S. Hunt, A. Coster, A technique for calculating meteor plasma density and meteoroid mass from radar head echo scattering, Icarus 168 (1) (2004) 43-52.

[46] A. Pellinen-Wannberg, The eiscat meteor-head method - a review and recent observations, Atmospheric Chemistry and Physics 4 (2004) 649655 .

[47] S. J. Ostro, Planetary radar astronomy, Rev. Mod. Phys. 65 (1993) 1235-1279. doi:10.1103/RevModPhys.65.1235. URL http://link.aps.org/doi/10.1103/RevModPhys .65.1235

[48] A. Virkki, K. Muinonen, A. Penttilä, Inferring asteroid surface properties from radar albedos and circular-polarization ratios, Meteoritics \& Planetary Science 49 (1) (2014) 86-94. doi:10.1111/maps.12161.

[49] M. Gritsevich, V. Vinnikov, T. Kohout, J. Tóth, J. Peltoniemi, L. Turchak, J. Virtanen, A comprehensive study of distribution laws for the fragments of Košice meteorite, Meteoritics \& Planetary Science 49 (3) (2014) 328-345.

[50] A. Ern, J.-L. Guermond, Theory and practice of finite elements, Springer Science \& Business Media, 2004.

[51] J. R. Phillips, J. K. White, A precorrected-FFT method for electrostatic analysis of complicated 3-D structures, IEEE Trans. Computer-Aided Design of Integrated Circuits and Systems 16 (1997) 1059-1072. 